\title{
Nível de atividade física, autonomia funcional e qualidade de vida em idosas ativas e sedentárias
}

\author{
Level of physical activity, functional autonomy and quality of life in \\ sedentary and active elderly women
}

\author{
Nelyse de Araújo Alencar ${ }^{[a]}$, José Vitorino de Souza Júnior ${ }^{[b]}$, Jani Cleria Bezerra Aragão ${ }^{[c]}$, \\ Márcia de Assunção Ferreira ${ }^{[\mathrm{d}]}$, Estélio Dantas ${ }^{[\mathrm{e}]}$
}

[a] Enfermeira, Mestre em Ciências da Motricidade Humana (PROCIMH-UCB/RJ), Juazeiro do Norte, CE - Brasil, e-mail: nelyse@bol.com.br / nelysearaujo@hotm

[b] Fisioterapeuta, Mestre em Saúde da Criança e do Adolescente, Especialista em Fisioterapia Cardiorrespiratória e Traumatoortopédica, professor da Faculdade de Fisioterapia Leão Sampaio (FALS), Juazeiro do Norte, CE - Brasil.

[c] Professora Doutora do Programa de Pós-Graduação Stricto Sensu em Ciências da Motricidade Humana (PROCIMH-UCB/RJ), Rio de Janeiro, RJ - Brasil, e-mail: jani.cleria@cobrase.org.br

[d] Professora, Doutora em Enfermagem, Escola de Enfermagem Anna Nery, Universidade Federal do Rio de Janeiro (UFRJ), Rio de Janeiro, RJ - Brasil, e-mail: marciadeaf@ibest.com.br

[e] Professor Doutor do Programa de Pós-Graduação Stricto Sensu em Ciências da Motricidade Humana (PROCIMH-UCB/RJ), Rio de Janeiro, RJ - Brasil, e-mail: estelio@cobrase.org.br

\section{Resumo}

Introdução: $O$ envelhecimento é um processo contínuo durante o qual ocorre declínio progressivo de todos os processos fisiológicos. Objetivo: O objetivo do presente estudo foi analisar os níveis de atividade física, autonomia funcional e qualidade de vida em idosas ativas e sedentárias. Método: A amostra foi constituída de 30 mulheres (15 sedentárias e 15 ativas), com idade igual ou superior a 60 anos. Para determinar o nível de atividade física, utilizou-se a versão do Questionário Baecke Modificado para Idosos; e a autonomia funcional foi avaliada pela bateria de testes do Grupo de Desenvolvimento Latino-Americano para a Maturidade (GDLAM); e a qualidade de vida, por meio do questionário WHOQOL-OLD. O tratamento estatístico foi composto por análise descritiva e inferencial. Os dados foram analisados pelo SPSS ${ }^{\circledR}$, versão 13.0. O nível de significância e erro estatístico considerado foi de $5 \%(p<0,05)$. Resultados e discussão: Pela análise dos resultados pôde-se observar que os níveis de atividade física encontrados nas idosas ativas foi maior que os das sedentárias, repercutindo nos melhores resultados apresentados nos testes de autonomia funcional, apesar de ambas serem classificadas com 
IG fraco. Em relação à qualidade de vida, constatamos não haver associação a melhores níveis quando comparamos ambos os grupos. Considerações finais: Recomenda-se que seja realizada uma intervenção por meio de um programa de treinamento de físico mais intenso, envolvendo maior quantidade de indivíduos mais bem condicionados para melhor verificar as possíveis alterações das variáveis do estudo realizado.

Palavras-chave: Atividade física. Autonomia. Qualidade de vida. Idoso.

\begin{abstract}
Introduction: The aging is a continuous process during which occurs the progressive decline in all physiological processes. Objective: The objective of this study was to analyze the levels of physical activity, functional autonomy and quality of life in active and sedentary elderly women. Method: The sample consisted of 30 women (15 sedentary and 15 active), aged equal or over 60 years-old. To determine the level of physical activity, it was used the version of the Modified Baecke Questionnaire for Elderly People; the functional autonomy was assessed by the battery of tests from the Group of Latin American Development to Maturity (GDLAM); and quality of life by WHOQOL-OLD. The statistical treatment consisted of descriptive and inferential analysis. The data were analyzed using SPSS ${ }^{\mathrm{TM}}, 13.0$ version. The level of significance and statistical error considered was set at $5 \%(p<0.05)$. Results and discussion: By examining the results it could be observed that the levels of physical activity found in the active elderly women was higher than the sedentary ones, reflecting the best results in the tests of functional autonomy $(p<0.05)$, although both are classified as low GI. Regarding the quality of life, we found no association at higher levels when comparing both groups. Final considerations: It is recommended, therefore, that an intervention is made through a more intense physical training program, with larger numbers of individuals more well conditioned to better evaluate the possible alterations of the variables from this study.
\end{abstract}

Keywords: Physical activity. Autonomy. Quality of life. Elderly.

\title{
Introdução
}

O envelhecimento populacional é um fenômeno relativamente novo em todo mundo. Conforme dados do Censo Demográfico do IBGE (1), há cerca de 10 milhões de pessoas na população brasileira com idade superior a 65 anos (2). Ainda segundo dados do IBGE, no ano de 2020 o Brasil terá a sexta população mundial em números absolutos de idosos (3).

Com o crescimento mundial da população idosa, a preocupação em relação à capacidade funcional vem surgindo como novo destaque para a estimativa da saúde desse segmento etário. Esse aumento gera maior probabilidade de ocorrência de doenças crônicas e, com isso, o desenvolvimento de incapacidades associadas ao envelhecimento (4). Um aumento das limitações das atividades de vida diária está diretamente associado ao aumento de comorbidades nos idosos (5).

O treinamento físico tem sido considerado um importante esquema terapêutico não farmacológico no tratamento e na prevenção de eventos cardiovasculares, assim como para portadores de fatores de risco com maior propensão ao desenvolvimento de doenças cardíacas (6).

A qualidade de vida é um fator diretamente ligado a esse contexto, sendo um dos responsáveis pelo aumento ou pelo decréscimo na longevidade da população. A preocupação em manter hábitos que garantam uma velhice saudável marca uma nova etapa de conscientização. As atividades físicas são importantes para que se atinja o padrão desejado em certos aspectos da qualidade de vida e autonomia funcional nesses indivíduos (7).

Apesar dos benefícios da prática de atividade física serem amplamente divulgados, poucos são os que realizam tais atividades regularmente, principalmente o subgrupo de idosos. As pesquisas que avaliam 
os níveis de atividade física, autonomia funcional e qualidade de vida na população de idosos no Brasil estão crescendo cada vez mais diante do visível envelhecimento da população, porém pesquisas dessa magnitude ainda são escassas nos municípios citados.

Dentro desse contexto, estudos que busquem atualizar o conhecimento sobre a problemática do envelhecimento favorecem a aquisição de informações e promovem discussões sobre o tema, contribuindo assim para uma formação de profissionais competentes no cuidado com o idoso, em suas diferentes nuances.

Espera-se, assim, incentivar os profissionais de saúde e gestores à implementação de políticas públicas que atendam às necessidades dos idosos, no planejamento de ações voltadas para promoção da saúde, reforçando a divulgação dos benefícios da prática regular da atividade física, chamando a atenção para a sua importância como fator predominante de proteção e promoção à saúde, como forma não só de prevenção primária das doenças crônicas, mas também da prevenção secundária em pacientes com doenças cardiovasculares e outras enfermidades, no intuito de envolver a comunidade idosa na prática de tais atividades; além de chamar a atenção para a importância da inserção do profissional de Educação Física na Estratégia Saúde da Família.

Baseado no exposto, a questão cerne do estudo problematiza-se da seguinte forma: quais os níveis de atividade física, autonomia funcional e qualidade de vida em idosas ativas e idosas sedentárias? Visando a encontrar resultados que contribuíssem para resolução dessa problemática, objetivou-se realizar uma análise comparativa dos referidos níveis.

\section{Materiais e métodos}

A pesquisa caracterizou-se como quase-experimental do tipo expost facto, descritiva e comparativa. A amostra foi caracterizada como finita, não probabilística intencional e escolhida por conveniência, mediante aceitação em participar do estudo, por meio da assinatura do Termo de Consentimento Livre e Esclarecido, sob protocolo n. 0159/2008 do Comitê de Ética e Pesquisa da Universidade Castelo Branco, RJ, de acordo com a Resolução n. 196/96. As participantes apresentavam idade igual ou superior a 60 anos, eram praticantes e não praticantes de atividade física regular (há pelo menos três meses) e cadastradas em unidades básicas de Saúde de Família das cidades de Crato e Juazeiro do Norte, CE.

Como critério de inclusão, as participantes deveriam ser cadastradas nas unidades do Programa Saúde da Família dos referidos municípios, ser do sexo feminino e ter idade igual ou superior a 60 anos. Foram considerados critérios de exclusão: indivíduos do sexo masculino; idosa com qualquer tipo de enfermidade aguda ou crônica que pudesse comprometer ou se tornar um fator de impedimento para os testes realizados; as que faziam uso de medicamentos que causassem distúrbios de atenção ou as não cadastradas nas respectivas unidades de saúde.

A amostra foi sorteada aleatoriamente de um universo de 120 idosas e dividida em um grupo de 15 idosas sedentárias, ou seja, que não praticavam nenhum tipo de atividade física regular, e um grupo de 15 idosas ativas que realizavam caminhada de intensidade leve, três vezes na semana, por 30 minutos, há três meses.

Para determinar o nível de atividade física, foi utilizada a versão do Questionário Baecke Modificado para Idosos (QBMI) que avalia o nível de atividade física em três domínios específicos: tarefas realizadas em casa, atividades esportivas e atividades de lazer. Os escores são obtidos por meio de perguntas específicas e pela relação entre tipo, frequência e intensidade da atividade. Escores menores, nesse instrumento, representam menor nível de atividade física realizado pelo sujeito (8).

Para a avaliação da Autonomia Funcional, as idosas foram submetidas a uma bateria composta por cinco testes adotados no protocolo de avaliação funcional do Grupo de Desenvolvimento Latino-Americano para a Maturidade (GDLAM): caminhar 10 metros - C10 m (9); levantar-se da posição sentada - LPS (10); levantar-se da posição decúbito ventral - LPDV (11); levantar-se da cadeira e locomover-se pela casa - LCLC (12). Todos esses testes são utilizados para o cálculo do Índice de GDLAM (IG). O tempo desses testes é 
calculado em segundos. Escores menores, nesse instrumento, representam melhor nível de autonomia funcional e vice-versa. O IG é calculado da seguinte forma:

$$
\mathrm{IG}=\frac{[(\mathrm{C} 10 \mathrm{~m}+\mathrm{LPS}+\mathrm{LPDV}) \times 2] \times \mathrm{LCLC}}{3}
$$

Na Tabela 1 são apresentadas as classificações da avaliação da autonomia, por meio do protocolo GDLAM, obtido por quartis (13).

Tabela 1 - Padrão de Avaliação da Autonomia Funcional do Protocolo GDLAM

\begin{tabular}{lccccc}
\hline $\begin{array}{l}\text { Testes } \\
\text { Classif. }\end{array}$ & $\begin{array}{l}\text { C10m } \\
(\mathbf{s e g})\end{array}$ & $\begin{array}{c}\text { LPS } \\
(\mathbf{s e g})\end{array}$ & $\begin{array}{c}\text { LPDV } \\
(\mathbf{s e g})\end{array}$ & $\begin{array}{c}\text { LCLC } \\
(\mathbf{s e g})\end{array}$ & $\begin{array}{c}\text { IG } \\
(\text { escores })\end{array}$ \\
\hline Fraco & $+6,89$ & $+11,47$ & $+4,51$ & $+43,00$ & $+28,54$ \\
Regular & $6,89-6,23$ & $11,47-9,86$ & $4,51-3,48$ & $43,00-38,69$ & $28,54-25,25$ \\
Bom & $6,22-5,58$ & $9,85-8,35$ & $3,47-2,78$ & $38,68-34,78$ & $25,24-22,18$ \\
Muito bom & $-5,58$ & $-8,35$ & $-2,78$ & $-34,78$ & $-22,18$ \\
\hline
\end{tabular}

Legenda: C10m = caminhar 10 metros; LPS = levantar da posição sentada; LPDV = levantar da posição de decúbito ventral; LCLC = levantar da cadeira e locomover-se pela casa; IG = índice GDLAM.

Para avaliar a qualidade de vida utilizou-se o questionário WHOQOL-OLD, que consiste de 24 facetas em uma escala de Likert atribuídos a seis facetas (domínios): "Funcionamento do sensório" (FS), "Autonomia" (AUT), "Atividades Passadas, Presentes e Futuras" (PPF), "Participação Social" (PSO), "Morte e Morrer" (MEM), e "Intimidade" (INT), (14).

A análise dos dados foi realizada utilizando-se o pacote estatístico SPSS ${ }^{\circledR}$, versão 13.0, os resultados da análise estatística descritiva são apresentados com média, desvio padrão, mínima e máxima e a análise estatística inferencial foi desempenhada pelo teste $t$ de Student, objetivando verificar a existência de diferenças significativas entre os testes de ambos os grupos. Para todos os procedimentos, adotou-se um nível de confiança de $5 \%(p<0,05)$.

\section{Resultados}

As tabelas a seguir apresentam a estatística descritiva e inferencial para os resultados do nível de atividade física, protocolo de GDLAM e qualidade de vida do grupo de idosas ativas (A) e sedentárias (S).

Para avaliar o nível de atividade física, soma-se os valores, entendendo que a valência é diretamente proporcional à sua respectiva classificação, isto é, à medida que o valor de pontuação (escore) cresce, a classificação aproxima-se de ativa e vice-versa (Tabela 2).

As Tabelas a seguir apresentam os resultados dos testes em segundos de avaliação da autonomia funcional e da qualidade de vida da amostra. Para avaliarmos a autonomia funcional, entendemos que a valência é inversamente proporcional aos resultados dos testes, ou seja, à medida que os valores dos testes em segundos crescem, a classificação da autonomia diminui e vice-versa (Tabela 3). 
A qualidade de vida é analisada de acordo com os escores das seis facetas (domínios). Para todas as facetas o escore dos valores possíveis pode oscilar de 4 a 20. Os escores dessas seis facetas são combinados para produzir um escore geral (Qvg-old). Basicamente, escores altos representam uma alta qualidade de vida, escores baixos representam uma baixa qualidade de vida (Tabela 4).

Tabela 2 - Estatística descritiva e inferencial do nível de atividade física(QBMI)

\begin{tabular}{|c|c|c|c|c|c|c|c|c|c|}
\hline \multirow{2}{*}{ Atividades } & \multicolumn{2}{|c|}{ média } & \multicolumn{2}{|c|}{$\mathrm{dp}$} & \multicolumn{2}{|c|}{ mín. } & \multicolumn{2}{|c|}{ máx. } & \multirow{2}{*}{$p$-valor } \\
\hline & $\mathbf{A}$ & S & A & $\mathrm{S}$ & $\mathbf{A}$ & S & A & $\mathrm{S}$ & \\
\hline Domésticas & 1,7 & 1,3 & 0,3 & 0,4 & 1,1 & 0,7 & 2,3 & 1,9 & 0,018 \\
\hline Esportivas & 0,0 & 0,0 & 0,0 & 0,0 & 0,0 & 0,0 & 0,0 & 0,0 & - \\
\hline Lazer & 1,4 & 0,3 & 1,6 & 0,4 & 0,3 & 0,0 & 6,19 & 1,19 & 0,02 \\
\hline Escore & 3,2 & 1,7 & 1,7 & 0,8 & 1,4 & 0,7 & 9,3 & 3,0 & 0,007 \\
\hline
\end{tabular}

Legenda: $\mathrm{A}=$ ativas, $\mathrm{S}=$ sedentárias, $\mathrm{dp}=$ desvio padrão, mín=mínima, max=máxima.

Tabela 3 - Estatística descritiva e inferencial da autonomia funcional (GDLAM)

\begin{tabular}{|c|c|c|c|c|c|c|c|c|c|}
\hline \multirow{2}{*}{ Testes } & \multicolumn{2}{|c|}{ média } & \multicolumn{2}{|c|}{$\mathrm{dp}$} & \multicolumn{2}{|c|}{ mín. } & \multicolumn{2}{|c|}{ máx. } & \multirow{2}{*}{$p$-valor } \\
\hline & A & $\mathrm{S}$ & A & S & A & $\mathrm{S}$ & A & S & \\
\hline C10 & 7,9 & 16,3 & 2,2 & 5,7 & 5,6 & 8,1 & 13,7 & 32,3 & 0,000 \\
\hline LPS & 12,3 & 17,5 & 3,0 & 4,9 & 6,7 & 9,4 & 19,5 & 24,6 & 0,002 \\
\hline LPDV & 3,5 & 9,8 & 1,0 & 11,3 & 2,3 & 2,1 & 5,8 & 48,5 & 0,04 \\
\hline LCLC & 21,7 & 39,2 & 7,9 & 15,1 & 10,6 & 29,5 & 55,7 & 57,8 & 0,00 \\
\hline IG & 28,9 & 36,4 & 5,0 & 13,3 & 23,2 & 38,3 & 38,3 & 78,5 & 0,05 \\
\hline
\end{tabular}

Legenda: $\mathrm{A}=$ ativas, $\mathrm{S}=$ sedentárias, $\mathrm{dp}=$ desvio padrão, mín=mínima, $\max =$ máxima, $\mathrm{C} 10 \mathrm{~m}$ = caminhar 10 metros; LPS = levantar da posição sentada; LPDV = levantar da posição de decúbito ventral; LCLC = levantar da cadeira e locomover-se pela casa; IG = índice GDLAM.

Tabela 4 - Estatística descritiva e inferencial da qualidade de vida (WHOQOL-OLD)

(continua)

\begin{tabular}{|c|c|c|c|c|c|c|c|c|c|}
\hline \multirow{2}{*}{ Domínios } & \multicolumn{2}{|c|}{ média } & \multicolumn{2}{|c|}{ dp } & \multicolumn{2}{|c|}{ mín. } & \multicolumn{2}{|c|}{ máx. } & \multirow{2}{*}{$p$-valor } \\
\hline & $\mathbf{A}$ & $\mathrm{S}$ & A & S & $\mathbf{A}$ & $\mathrm{S}$ & A & S & \\
\hline Dom 1 & 9,7 & 10,7 & 2,4 & 2,6 & 6,0 & 6,0 & 15,0 & 15,0 & 0,29 \\
\hline Dom 2 & 13,6 & 10,5 & 3,6 & 3,8 & 7,0 & 5,0 & 18,0 & 16,0 & 0,03 \\
\hline
\end{tabular}


Tabela 4 - Estatística descritiva e inferencial da qualidade de vida (WHOQOL-OLD)

(conclusão)

\begin{tabular}{|c|c|c|c|c|c|c|c|c|c|}
\hline \multirow{2}{*}{ Domínios } & \multicolumn{2}{|c|}{ média } & \multicolumn{2}{|c|}{ dp } & \multicolumn{2}{|c|}{ mín. } & \multicolumn{2}{|c|}{ máx. } & \multirow{2}{*}{$p$-valor } \\
\hline & A & S & $\mathbf{A}$ & S & A & $\mathrm{S}$ & $\mathbf{A}$ & S & \\
\hline Dom 3 & 13,8 & 11,8 & 2,4 & 2,2 & 9,0 & 7,0 & 18,0 & 16,0 & 0,02 \\
\hline Dom 4 & 15,4 & 13,9 & 2,2 & 2,4 & 12,0 & 9,0 & 9,0 & 18,0 & 0,09 \\
\hline Dom 5 & 12,6 & 14,3 & 4,4 & 11,9 & 4,0 & 10,0 & 18,0 & 4,0 & 0,17 \\
\hline Dom 6 & 14,5 & 14,4 & 3,7 & 1,6 & 4,0 & 10,0 & 20,0 & 17,0 & 0,95 \\
\hline Qvg-old & 13,2 & 12,6 & 1,9 & 1,3 & 11,6 & 10,0 & 16,3 & 15,0 & 0,19 \\
\hline
\end{tabular}

Legenda: $\mathrm{A}=$ ativas, $\mathrm{S}=$ sedentárias, $\mathrm{dp}=$ desvio padrão, mín=mínima, max=máxima, Dom1=Funcionamento do sensório, Dom2=Autonomia, Dom3=Atividades Passadas, Presentes e Futuras, Dom4=Participação Social, Dom5=Morte e Morrer, Dom6=Intimidade, Qvg-old=qualidade de vida geral.

\section{Discussão}

É fato que as atividades físicas são de grande importância para a manutenção da boa saúde dos idosos, e que eles apresentam uma amplitude muito grande de níveis de atividade física. Desta forma, o método de avaliação do nível de atividade física por meio do QBMI mostrou-se capaz de fazer esta análise, como esperado (15).

Na Tabela 2, observa-se que os escores do QBMI encontrados em ambos os grupos (ativas $=3,2$; sedentárias $=1,7$ ) ainda estão muito aquém dos pontos encontrados em estudos semelhantes que envolveram idosos. Toma-se como exemplo o estudo de Miyasike da Silva (16), realizado com 61 idosos divididos em três grupos, em que foi encontrada uma média de 3,19 pontos para um grupo de idosos sedentários e em outros dois grupos de idosos fisicamente ativos, médias de 8,53 e 7,82 pontos. Também no estudo de Menezes (17), as médias encontradas foram superiores às médias desta pesquisa, sendo que para idosos participantes de um grupo de atividade física supervisionada a média foi de 12,73, enquanto que para os que não apresentavam tal participação foi de 7,48. Porém, na presente pesquisa, quando comparamos os dois grupos, notamos que houve uma diferença significativa nos resultados das atividades realizadas e no escore total ( $p=0,007)$, ou seja, as idosas praticantes de atividade física regular, apesar de apresentarem escores menores do que o esperado, ainda obtiveram resultados melhores que as sedentárias. Esse resultado corrobora o encontrado por Gobbi et al. (18), segundo o qual indivíduos que realizam somente atividades da vida diária não possuem o mesmo NAF quando comparado com pessoas engajadas em programas de atividade física regular.

A prevalência de baixos níveis de atividade física também foi observada por Kuhnen (19) em um estudo realizado em Florianópolis com idosos, em que aproximadamente $40 \%$ deles apresentaram níveis insuficientes, confirmando os resultados encontrados na presente pesquisa.

Dados de pesquisas realizadas na Alemanha e em Singapura afirmam que a atividade física é um pré-requisito para um envelhecimento com sucesso (20). Berlezi et al. (21) demonstraram, em estudo realizado com idosas, que a flexibilidade e resistência muscular localizada podem ser melhoradas com a prática de atividade física regular.

Analisando a Tabela 3, verifica-se que as idosas ativas obtiveram melhores resultados, havendo diferença estatística significante $(p<0,05)$ para todos os testes realizados, mas quando os valores são comparados aos valores do protocolo de GDLAM, observamos que os dois grupos obtiveram a classificação do IG como fracos. Notou-se que nos testes C10, LPS, LCLC e LPDV as idosas ativas apresentaram resultados melhores e significantes $(p<0,05)$ em relação ao outro grupo. Esses testes avaliam a agilidade e o equilíbrio do idoso 
executante, tornando-se, assim, um importante instrumento de avaliação da autonomia em desempenhar atividades na vida diária, e tem sido amplamente utilizado não só na avaliação de idosos assintomáticos (22-23), mas também de idosos portadores de doenças crônicas (24). Esses resultados estão de acordo com Dantas e Vale (25).

O fator mais importante para se avaliar a dependência e também o risco de mortalidade do indivíduo idoso é sua capacidade para desempenhar as atividades da vida diária (AVD), que está associada à autonomia funcional (26). Segundo Ramos et al. (27), idosos com dependência para 7 ou mais têm três vezes mais risco de mortalidade do que indivíduos independentes, sendo que a dependência nas AVD é um fator que pode ser mutável com prevenção e reabilitação. Além disso, os transtornos causados pela perda progressiva da autonomia refletem-se nos diversos domínios na vida dos gerontes, provocando consequências, como uma motricidade desequilibrada e precária (28).

Ao analisar os dados da Tabela 4, classificamos os indivíduos ativos com média do Qvg-old igual ou superior a 13 como sendo detentores de qualidade de vida suficiente, enquanto aqueles que apresentaram condições inferiores foram classificados com Qvg-old insuficiente. Para os indivíduos sedentários a média do Qvg-old encontrado foi de 12,6; sendo assim, observamos na amostra que 46\% dos indivíduos ativos apresentaram níveis iguais ou acima de 13, sobrepondo-se aos $40 \%$ dos indivíduos sedentários que obtiveram níveis superiores a 13, demonstrando que o fato de praticar atividade física regular não esteve associado a melhores níveis de qualidade de vida nessa amostra. Isso fica bem evidenciado quando analisamos a significância dos seis domínios em ambos os grupos (Qvg-old, p > 0,05). Esses dados corroboram o estudo de Vale et al. (29), o qual usou o formulário WHOQOL-100 para avaliar a qualidade de vida em grupos de ativos e sedentários, pois nesse estudo também não foi constatada diferença significante de qualidade de vida em ambos os grupos. Todavia, na avaliação dos resultados na pesquisa de Castro (30), na comparação da qualidade de vida, utilizando o WHOQOL-OLD, em mulheres ativas e sedentárias, observamos que os domínios 2 e 3 das idosas ativas apresentaram valores significantes $(p<0,001)$ em relação ao grupo de sedentárias. O mesmo foi observado na presente pesquisa, ao analisarmos os resultados individuais dos domínios $2(p=0,03)$ e $3(p=0,02)$, conforme a Tabela 4.

\section{Conclusão}

A presente pesquisa pôde inferir que os níveis de atividade física encontrados nas idosas ativas foi maior que as sedentárias, repercutindo em efeitos evidentes nos melhores resultados apresentados nos testes de autonomia funcional, apesar de ambas serem classificadas com IG fraco. Conclui-se que os indicadores de autonomia para realizar as atividades da vida diária pelo protocolo GDLAM atingiram índices insatisfatórios nos dois grupos, reforçando a premissa que o envelhecimento associado ao sedentarismo provoca diminuição da capacidade funcional. Em relação à qualidade de vida, constatamos não haver diferença significativa entre a prática de atividade física e melhores níveis de qualidade de vida quando comparamos o grupo de ativas com o de sedentárias.

Recomenda-se, assim, que seja realizada uma intervenção por meio de um programa de treinamento de físico mais intenso, envolvendo maior quantidade de indivíduos melhor condicionados para verificar de modo mais eficaz as possíveis alterações nas variáveis do estudo realizado. Enfatizamos que o conhecimento sobre o processo de senescência e senilidade pelos profissionais de saúde que lidam com idosos é de crucial relevância, devendo-se trabalhar com esse grupo sempre buscando respeitar suas limitações, enfatizando seu potencial remanescente e sua capacidade para o autocuidado. Levando ainda em consideração que cada idoso possui a sua história de vida, diferente de qualquer outra e o que pode significar qualidade de vida e bem-estar para ele pode ser diferente do significa para o profissional da saúde.

\section{Referências}

1. Fundação Instituto Brasileiro de Geografia e Estatística - IBGE. Senso 2000. [online] 2000. [acesso em 20 jan. 2007]. Disponível em http://www.ibge.gov.br 
2. Fleck SJ, Figueira Jr A. Treinamento de força para fitness e saúde. São Paulo: Phorte; 2003.

3. Fundação Instituto Brasileiro de Geografia e Estatística - IBGE. Senso 2004. [online] 2004. [acesso em 20 jan. 2007]. Disponível em http://www.ibge.gov.br

4. Rosa TEC, Benício MHD, Latorre MRDO, Ramos LR. Fatores determinantes da capacidade funcional entre idosos. Rev Saúde Pública. 2003;37(1):40-8.

5. Calasans PA, Alouche SR. Correlação entre o nível cognitivo e a independência funcional após AVE. Rev Bras Fisioter. 2004;8(Suppl 2):105-9.

6. Sacilotto MC, Del Grossi RT, Sirol FN, Pessott ER, Sakabe Di, Silva E. Relação da freqüência cardíaca e da potência no pico do teste ergométrico e no nível do limiar de anaerobiose de homens de meia-idade saudáveis e de hipertensos. Fisioter Mov. 2007;20(4):43-53.

7. Matsudo, SMM. Envelhecimento \& atividade física. Londrina: Midiograf; 2001.

8. Voorrips LEA, Ravelli ACJ, Dongelmans PCA, Deurenberg P, Van SWA. A physical activity questionnaire for the elderly. Med Sci Sports Exerc. 1991;23(8):974-9.

9. Sipilä S, Multanen J, Kallinen M, Era P, Suominen H. Effects of strength and endurance training on isometric muscle strength and walking speed in elderly women. Acta Physiol Scand. 1996;156(4):457-64.

10. Guralnik JM, Simonsick EM, Ferrucci L, Glynn RJ, Berkman LF, Blazer DG, et al. A short physical performance battery assessing lower extremity function: association with self-reported disability and prediction of mortality and nursing. J Gerontol. 1994;49(2):M85-94.

11. Alexander NB, Ulbrich J, Raheja A, Channer D. Rising from the floors in older adults. J Am Geriatr Soc. 1997;45(5):564-9.

12. Andreotti Ra, Okuma SS. Validação de uma bateria de testes de atividades da vida diária para idosos fisicamente independentes. Rev Paul Educ Fís. 1999;13(1):46-66.

13. Vale RGS, Novaes JS, Dantas EHM. Efeitos do treinamento de força e de fexibilidade sobre a autonomia de mulheres senescentes. Rev Bras Ciênc Mov. 2005;13:33-40.

14. Fleck, MPA, Chachamovich E, Trentini CM. WHOQOL-OLD Project: method and focus group results in Brazil. Revista Saúde Pública [online]. 2003 [cited 2007 Mar. 9];37(6):793-9. Available from: http://www.scielosp.org/ scielo.php

15. Nascimento CMC. Nível de atividade física e principais barreiras percebidas entre idosos do bairro Vila Alemã Rio Claro/SP. Rev Educ Física. 2008;9(1):109-18.

16. Miyasike SV. Mobilidade de idosos em ambiente doméstico: efeitos de um programa de treinamento específico [monografia]. Rio Claro: Instituto de Biociências, Universidade Estadual Paulista, Rio Claro; 2000.

17. Menezes E. Questionário de Baecke modificado para idosos: programas de atividades físicas supervisionadas garantem maiores níveis de atividade física [monografia]. Instituto de Biociências, Universidade Estadual Paulista; 2002.

18. Gobbi S, Oliani MM, Lopes AG, Coutinho GF, Bucken LT, Costa JLR. Validade do teste de andar 6 minutos para idosas em relação ao tempo de fadiga. Rev Bras Ativ Fís Saúde. 2006;11(1):13-8.

19. Kuhnen P, Lopes AP, Benedetti TRB. Recursos econômicos atividades físicas de idosos de Florianópolis - SC. Rev Bras Ativ Fís Saúde. 2006;11(1):32-41.

20. Lehr U. A revolução da longevidade: impacto na sociedade, na família e no indivíduo. Est Interdiscipl Envelhec. 1999(1):7-35. 
21. Berlezi EM, Rosa PV, Souza ACA, Schneider RH. Comparação antropométrica e do nível de aptidão física de mulheres acima de 60 anos praticantes de atividade física regular e não praticantes. Rev Bras Geriat Gerontol. 2006;9(3).

22. Paula RH, Vale RGS, Batista LA, Oliveira, CG, Shung K, Estélio, HM. Efeitos da autonomia funcional de idosos sobre a fadiga muscular. Fisioterapia Brasil. 2008;9(1):33-8.

23. Pereira FF, Monteiro N, Vale RGS, Gomes ALM, Novaes JS, Faria AG, et al. Efecto del entrenamiento de fuerza sobre la autonomía funcional en mujeres mayores sanas. Rev Esp Geriat Gerontol. 2007;42(6):319-24.

24. Boechat F, Vale RGS, Dantas EHM. Evaluación de la autonomía funcional de personas mayores com EPOC mediante el protocolo GDLAM. Rev Esp Geriat Gerontol. 2007;42(4):251-3.

25. Dantas EHM, Vale RGS. Protocolo GDLAM de avaliação da autonomia funcional. Fitness Perfomance J. 2004;3(3):175-83.

26. Guimarães AC, Rocha CAQC, Gomes ALM, Cader SA, Dantas EHM. Efeitos de um programa de atividade física sobre o nível de autonomia de idosos participantes do programa de saúde da família. Fitness Perfomance J. 2008;7(1):5-9.

27. Ramos LR, Simies E, Albert MS. Dependence on daily living and cognitive impairment strongly predicted mortality among urban elderly residents in Brazil: a two-year follow up. J Am Geriat Soc. 2001;49:1168-75.

28. Aragão JCB, Dantas EHM, Dantas BHA. RML e autonomia. Fitness Performance J. 2002;1(3):29-38.

29. Valle RGS, Barreto ACG, Novaes JS, Dantas EHM. Efeitos do treinamento resistido na força máxima, na flexibilidade e na autonomia funcional de mulheres idosas. Rev Bras Cineantropom Desempenho Hum. 2006;8(4):52-8.

30. Castro JC. Efeitos de distintos programas de atividade física sobre os níveis de qualidade de vida e de depressão em idosas [dissertação]. Rio de janeiro: Universidade Castelo Branco; 2008.

Recebido: 28/04/2009

Received: 04/28/2009

Aprovado: 18/03/2010

Approved: 03/18/2010

Revisado: 24/06/2010

Reviewed: 06/24/2010 